\title{
One-Dimensional Plasmon in an Atomic-Scale Metal Wire
}

\author{
Tadaaki Nagao, ${ }^{1, *}$ Shin Yaginuma, ${ }^{1}$ Takeshi Inaoka,${ }^{2}$ and Toshio Sakurai ${ }^{3}$ \\ ${ }^{1}$ Nano System Functionality Center, National Institute for Materials Science, 1-1 Namiki, Tsukuba, Ibaraki 305-0044, Japan \\ ${ }^{2}$ Department of Materials Science and Engineering, Iwate University, 4-3-5 Ueda, Morioka 020-8551, Japan \\ ${ }^{3}$ Institute for Materials Research, Tohoku University, 2-1-1 Katahira, Aoba-ku, Sendai 980-8577, Japan
}

(Received 21 June 2006; published 12 September 2006)

\begin{abstract}
We have measured one-dimensional (1D) plasmons in an atom wire array on the $\mathrm{Si}(557)$-Au surface by inelastic scattering of a highly collimated slow electron beam. The angular dependence of the excitation energy clearly indicates the strong 1D confinement and free propagation of the plasma wave along the wire. The observed plasmon dispersion is explained very well by a quantum-mechanical scheme which takes into account dynamic exchange-correlation effects, interwire interactions, and spin-orbit splitting of the $1 \mathrm{D}$ bands. Although the qualitative feature of the plasmon dispersion is reminiscent of that of a highdensity free-electron gas, we detected the substantial influence of electron correlation due to strong 1D confinement.
\end{abstract}

DOI: $10.1103 /$ PhysRevLett.97.116802

One-dimensional electron systems (1DESs) such as metallic atom wires are expected to sustain a unique collective charge-density excitation which can be called onedimensional (1D) plasmon, or wire plasmon. This excitation mode propagates only along the wire and strongly reflects the confined nature of the electron motion. Such a dimensionality effect will show up clearly in the energy dispersion and the linewidth of a plasmon. For example, 3D-type plasmons (bulk and surface plasmons) have finite energies at $q=0$ (q denotes momentum) [1], but the energies of $1 \mathrm{D}$ and $2 \mathrm{D}$ plasmons vanish at $q=0[2,3]$, since the restoring force for charge-density waves in low dimension vanishes in the long-wavelength limit. However, in spite of its broad interest, experimental observation of a plasmon in an atomic-scale metal wire has been lacking so far and its details remain unexplored.

Recently, Au-induced 1D chain structures on stepped $\operatorname{Si}(111)$ surfaces were found to comprise 1DESs with metallic electron densities [4-9]. Among them, Au-Si atom wires formed on the $\mathrm{Si}(557)$ surface possess two proximal and deep $(\sim 1 \mathrm{eV})$ electron pockets [5-9] and can be regarded as an ideal 1D metal. This system has been providing many intriguing topics such as spin-charge separation in a Tomonaga-Luttinger liquid, lattice distortion by Peierls instability, etc., which originate from the restricted 1D motion of electrons [4-8]. Moreover, a recent theoretical study by ab initio calculation has proposed a large spinorbit (SO) splitting in the 1D electronic band which results from the strong Au $6 p$ character of this 1D band [10].

In this Letter, we report the electron-energy-loss spectroscopy (EELS) of the plasmon in such a model 1D system on the $\mathrm{Si}(557)$ surface. The measured plasmon clearly shows strong one-dimensional and metallic characteristics which are expected from a 1D electron gas. We analyzed the observed plasmon dispersion by a quantummechanical scheme including the effects of dynamic exchange correlation (XC), interwire interactions, and SO splitting of the 1D bands. These results demonstrate that,
PACS numbers: 73.20.Mf, 71.45.Gm, 72.15.Nj, 73.63.Nm

although the Fermi velocity of the 1DES is very high, suggesting its free-electron nature, the dynamic electron correlation effects, in fact, significantly affect the plasmon due to the strong $1 \mathrm{D}$ confinement.

The plasmon measurements were performed in ultrahigh vacuum $\left(6 \times 10^{-11}\right.$ Torr base pressure) by use of EELS with a highly collimated slow electron beam [11]. The spectrometer can also be operated in the energy-filtered low-energy electron diffraction (LEED) mode with a typical resolution of $0.005 \AA^{-1}$ and momentum range of $|\mathbf{q}|<$ $2.5 \AA^{-1}$. An ordered array of the Au-induced 1D structure is formed on the terraces of the stepped $\operatorname{Si}(557)$ surface along the [110] direction [see Figs. 1(b) and 1(c)] by depositing 0.2 monolayer of $\mathrm{Au}$ onto the surface held at $800 \mathrm{~K}$. After the deposition, the sample was cooled to room temperature and its degree of order was checked by spot profile analysis in the high-resolution LEED mode (full width at half maximum of the LEED spots was $\approx$ $0.01 \AA^{-1}$ ).

Figure 1(a) shows an energy-filtered LEED pattern taken from the atomic wires on the Si(557) surface. The directions parallel $\left(\mathbf{q}_{\|}\right)$and perpendicular $\left(\mathbf{q}_{\perp}\right)$ to the atom wires are shown by arrows in this figure. The sharp Bragg spots and their spacing indicate a highly ordered periodic wire array with $19 \AA$ spacing [9]. We scanned the wave vector (momentum transfer in EELS) in wire-parallel $\left(\mathbf{q}_{\|}\right)$and wire-perpendicular $\left(\mathbf{q}_{\perp}\right)$ directions, as shown in Fig. 1, and measured EELS spectra at each position in the reciprocal space. Figure 2 shows two sets of the momentum-resolved EELS spectra taken in such a manner. In the wire-parallel direction [Fig. 2(a)], a single loss peak is clearly observed to disperse rapidly from the mid- to near-infrared region as a function of $q_{\|}\left(=\left|\mathbf{q}_{\|}\right|\right)$accompanied by monotonic linewidth broadening. On the other hand, in the $\mathbf{q}_{\perp}$ direction [Fig. 2(b)], no prominent feature was observed. This means that the observed excitation freely propagates only along the wires but not across the wires. These features are 
(a)

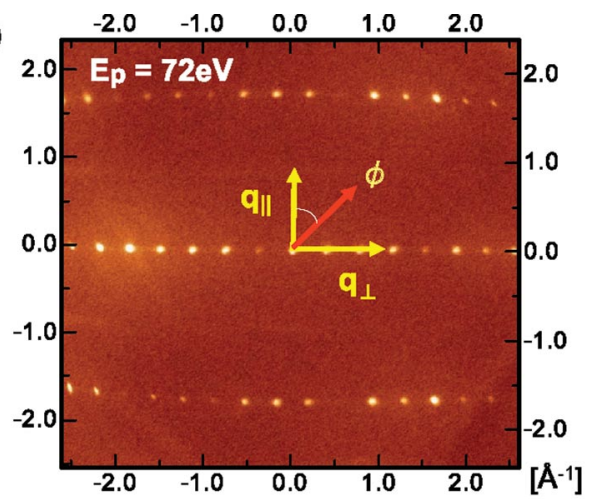

(b)

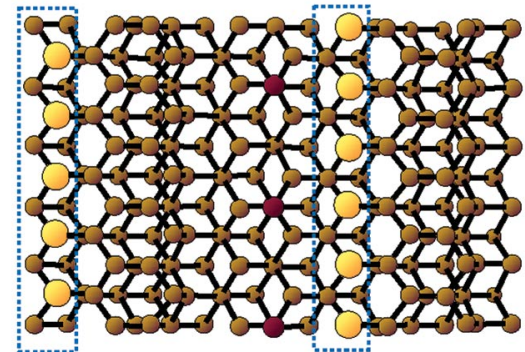

(c)

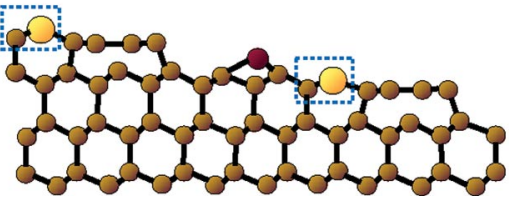

FIG. 1 (color). (a) High-resolution LEED pattern taken from the 1D structure self-assembled on the Si(557) surface. Scale markings are shown in $\left[\AA^{-1}\right]$ units. (b) Schematic illustration (top view) of the 1D structure. The larger (yellow) spheres represent $\mathrm{Au}$ atoms and the smaller (khaki) spheres Si atoms. $\mathrm{Si}$ adatoms are represented by smaller violet spheres. (c) Side view of the same structure. Au-incorporated 1D structure is indicated by blue dashed squares in (b) and (c).

essentially different from bulk, surface, and 2D plasmons, since they arise from electron motion confined in one dimension [1-3].

Figure 3 shows the energy dispersion curve determined from the peak positions of the losses in the EELS spectra in comparison with the theoretical curve described below. As clearly seen, the loss peak approaches zero as $q_{\|}$tends toward zero, which is the feature expected for plasmons in 1D and 2D. Below $q_{\|}=0.02 \AA^{-1}$ and $\hbar \omega=200 \mathrm{meV}$ [brown area in Fig. 3], each loss peak merges with the elastic peak and features a high Drude tail reflecting the strong metallic nature of the wires. Since the 1D plasmon exists only at the surface topmost layer, there should be no probing-depth dependence of the electron beam on the peak position; this is indeed the case, as we have checked by changing the incident energy from $E_{p}=26.2 \mathrm{eV}$ to $45.0 \mathrm{eV}$ [Fig. 3].

The 1D propagation of the excitation can be examined quantitatively by changing the azimuthal direction $\phi$ of the in-plane wave vector $\mathbf{q}$ from $\phi=0^{\circ}$ (along the wires) to

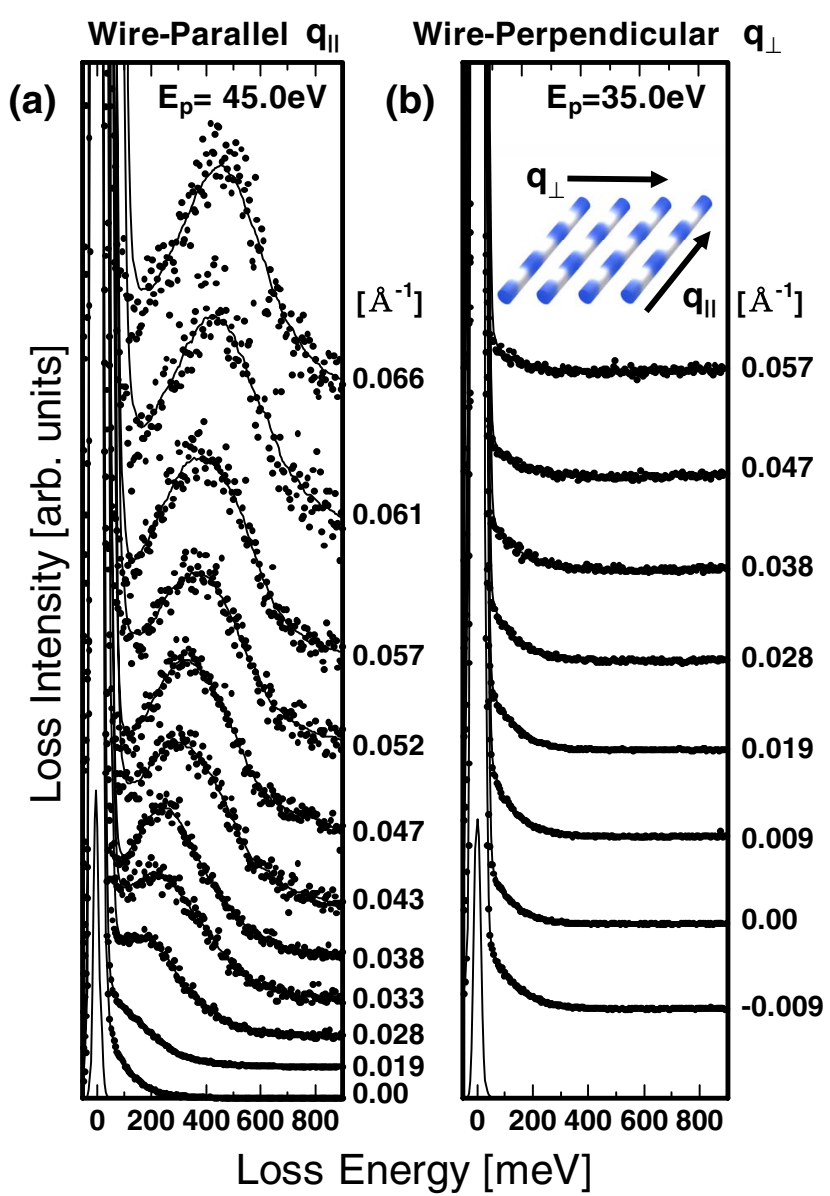

FIG. 2 (color online). Momentum-resolved EELS spectra for a series of wave numbers in the directions (a) parallel and (b) perpendicular to the atom wires. Wave numbers are indicated at the right side of each spectrum. The upper right inset shows the schematic illustration of the charge-density wave oscillations of the $1 \mathrm{D}$ plasmon.

$\phi=90^{\circ}$ (perpendicular to the wires). The frequency of the 1D plasmon follows the relation $\omega(q, \phi)=\omega(q \cos \phi, 0)$ [12]. This is observed as shown in Fig. 3. The double circles show data points for $\phi=45^{\circ}$ plotted versus the absolute value of $\mathbf{q}$. After replotting the data versus the component of $\mathbf{q}$ projected onto the wire direction, $q \cos \left(45^{\circ}\right)$ (double diamonds), they indeed fall again onto the same dispersion curve as for $\phi=0^{\circ}$. The above facts indicate that the observed loss is unambiguously assigned to the metallic 1D plasmon in the atom wire array.

Theoretically, dynamic XC effects were predicted to be stronger in a $1 \mathrm{D}$ plasma than in a $2 \mathrm{D}$ or $3 \mathrm{D}$ plasma. To examine this point quantitatively, we analyzed the data in the framework of a nonlocal dielectric-response theory with self-consistent local-field correction (LFC). We adopted the LFC developed by Singwi et al. [13] and analyzed the experimental data for the first time for 1D system. The XC effects can be described by the XC holes that make the electrons steer clear of each other within the 


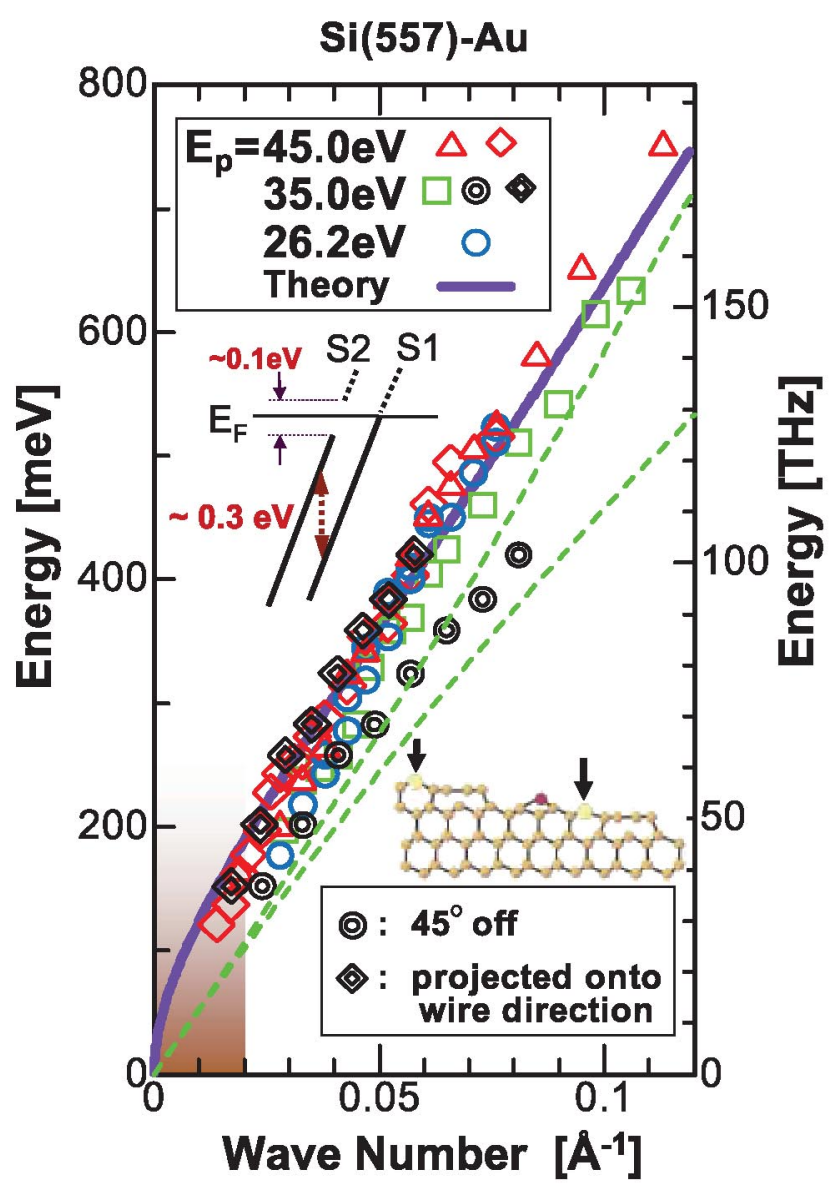

FIG. 3 (color). The energy dispersion curve determined from the peak positions of the losses in the EELS spectra in comparison with the theoretical curve described below. Precision in energy is roughly represented by the size of each data point, except for the ones below $q_{\|}=0.02 \AA^{-1}$ (brown area in the lower left). Below $0.02 \AA^{-1}$, each loss peak merges with the high metallic tail from the elastic peak and the precision becomes worse $( \pm 30 \mathrm{meV})$. The middle-left inset schematically shows the energy band diagram of two parallel bands S1 and S2 near the Fermi level $E_{F}$. The horizontal axis is the wave number and the vertical axis is the excitation energy. As shown in the lower-right inset (atomic arrangement), Au atoms constitute a chain structure without space inversion symmetry and will lead to spin-orbit splitting of the 1D band $[9,10]$. The area between the two green dashed curves corresponds to the single-particle excitation continuum.

distance $d_{\mathrm{xc}} \sim k_{F}{ }^{-1}$. Here $k_{F}$ signifies the Fermi wave number. This avoidance effect is considered to be more serious in $1 \mathrm{D}$ than in higher dimensions where the electrons have a greater choice of detours. The XC effects become more significant as $q_{\|}$becomes more comparable to $k_{F}$, i.e., $d_{\mathrm{xc}}{ }^{-1}$. Therefore, the theoretical fit in the large $q_{\|}$range accessible by EELS (inaccessible by optical methods) is necessary for making a quantitative evaluation of the XC effects. We also introduced an effective interwire interaction including the LFC [12]. In our analysis, the electron effective mass $m^{*}$ and the wire width $w$ are the fitting parameters. We used the available literature values of the silicon dielectric constant $\varepsilon_{\mathrm{Si}}=11.5$ [14] and $k_{F}=$ $0.41 \AA^{-1}[15]$ in our calculation.

The best theoretical fit to the experimental data is shown as the bold solid curve in Fig. 3. We can obtain markedly good agreement over the entire range of observed $q_{\|}$as wide as $0.01 \AA^{-1} \leq q_{\|} \leq 0.11 \AA^{-1}$. The parameters $m^{*}$ and $w$ are adjusted to take $m^{*}=0.60 m_{0}$ and $w=4 \AA$. Here $m_{0}$ stands for the free-electron mass. The wire width $w=4 \AA$ is reasonable considering that the $1 \mathrm{D}$ electronic bands originate from the Au-Si chains on the terraces $[9,10]$. The two proximal and parallel $1 \mathrm{D}$ bands are illustrated schematically in the middle-left inset in Fig. 3. The effective mass $m^{*}=0.60 m_{0}$ is also in good agreement with the value of $m^{*}=0.45 m_{0}$ determined by the photoelectron spectroscopy (PES) experiment $[5,6]$. This small deviation in the value of $m^{*}$ could arise from the difference in the portion of the band where the two methods focus their attention. In PES, $m^{*}$ reflects the band curvature near the band minimum, while in EELS, plasmons are composed of electronic transitions near the Fermi level $E_{F}$ and reflect the band dispersion near $E_{F}$. While the theoretical dispersion curve is very sensitive to changes in $m^{*}$, it is less sensitive to changes in $w$, since $w(4 \AA)$ is much shorter than the plasmon wavelength $2 \pi / q_{\|}$.

Our plasmon strongly depends on the structure of the two proximal $1 \mathrm{D}$ bands. If we assume that each of these two bands is metallic and/or twofold spin-degenerate, our theoretical fitting gives an unreasonably large $m^{*}$ value, i.e., $m^{*}>0.85 m_{0}$, and the calculated dispersion deviates significantly upward from the experimental one in a $q_{\|}$ range of $0 \AA^{-1}<q_{\|}<0.03 \AA^{-1}$. According to a recent proposal, the two parallel and proximal 1D bands are SO-split bands, because of its strong Au $6 p$ character and the loss of the space inversion symmetry of the chain structure $[9,10]$. If we accept this proposal, and assume one of the bands is insulating [16], we could indeed obtain very good agreement with the PES results in $m^{*}$ value as shown above. Therefore, our plasmon is likely to be assigned as an intraband plasmon in one of the SO-split bands with electron density $N_{1 \mathrm{D}}=k_{F} / \pi=0.13 \AA^{-1}=1.3 \times 10^{7} \mathrm{~cm}^{-1}$.

Although we have expected that the observed plasmon behaves as an ideal free-electron plasma, our quantitative analysis has revealed that the dynamic XC effects are, in fact, significant. The effective density parameter (electron density) of this system is as small as (as high as) $r_{s}{ }^{*}=$ $1 /\left(2 N_{1 \mathrm{D}} a_{B}{ }^{*}\right)=0.52$ for $m^{*}=0.45 m_{0}$ and $r_{s}{ }^{*}=0.70$ for $m^{*}=0.60 m_{0}$. Here the effective Bohr radius $a_{B}{ }^{*}$ is defined as $a_{B}{ }^{*}=\left\{\left(\varepsilon_{\mathrm{Si}}+1\right) / 2\right\}\left(m_{0} / m^{*}\right) \quad a_{B}$ (Bohr radius $\left.a_{B}=0.529 \AA\right)$. This indicates a very high average velocity of electrons, and, consequently, the electron-electron coupling is expected to be negligible. In spite of this fact, with an increase in $q_{\|}$, the XC effects start to redshift the plasmon frequency noticeably. If we switch off the XC by 


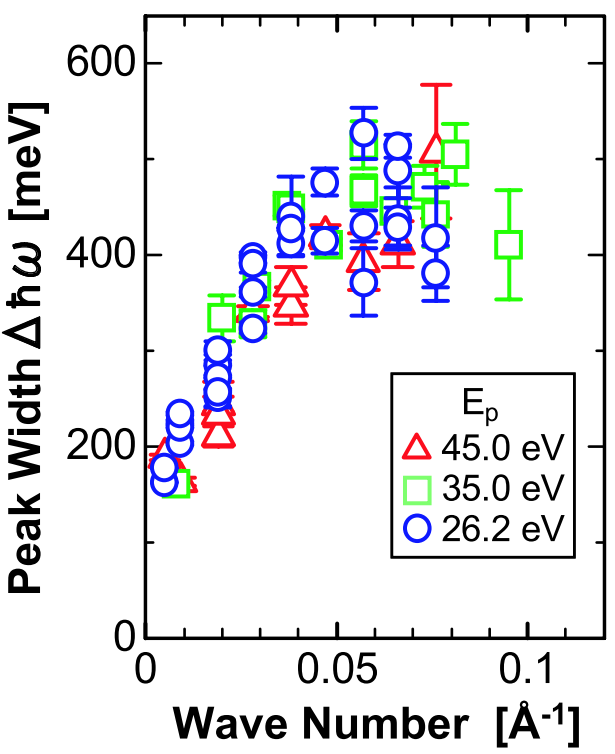

FIG. 4 (color online). Linewidth (lifetime) dispersion plotted against $q_{\|}$. The linewidth increases monotonically from $q_{\|}=$ $0 \AA^{-1}$ and saturates at around $q_{\|}=0.05 \AA^{-1}$. Such linewidth broadening could arise from the plasmon decay through two (and higher multiple) electron-hole pairs induced by Coulomb collision or electron-electron scattering as was previously observed in a strongly degenerate $2 \mathrm{DES}$ in metallic atom sheet $[2,3]$.

setting the LFC to zero, the plasmon energy at $q_{\|}=\frac{1}{4} k_{F}$, for example, undergoes a significant blueshift of as high as $\approx 9.4 \%$. Switching on the XC effects, the calculated plasmon dispersion agrees with the experimental one quite well when the $m^{*}$ value $\left(m^{*} \approx 0.60 m_{0}\right)$ is closer to that of the $\operatorname{PES}\left(m^{*} \approx 0.45 m_{0}\right)$. If we compare with the case of the $2 \mathrm{D}$ plasmon, for example, for a metallic atom sheet with even lower effective density $\left(r_{s}{ }^{*}=1.1\right.$ [17]), switching off the $\mathrm{XC}$ induces a blueshift of only $\approx 6 \%$ at $q_{\|}=\frac{1}{4} k_{F}$. This clearly demonstrates that the dynamic XC effects become more conspicuous in $1 D$ than in $2 D$, due to stronger electron confinement in 1D.

In the framework of the nearly free electron theory, the 1D plasmon should be free from decay by particle-hole pair creation up to a critical wave number $q_{\|, c}$ (Landau damping). In contrast to this picture, our data clearly show strong damping starting already from $q_{\|}=0$ [see Fig. 4]. The same type of damping was also observed for the $2 \mathrm{D}$ plasmon [2,3] and assigned as decay via Coulomb collision or electron-electron scattering [18]. This strongly suggests the importance of electron correlation effects also in the decay of the 1D plasmon.

For the first time, we measured the plasmon dispersion of atomic-scale wires prepared on a stepped silicon surface. A clear $1 \mathrm{D}$ and strong metallic nature, possibly associated with the Au-induced SO-split metallic 1D bands, was evidenced. Theoretical analysis combined with the wide- $q_{\|}$-range measurement revealed significant dynamic XC effects on the 1D plasmon in spite of its high electron density $\left(r_{s}{ }^{*}=0.5-0.7\right)$ and large Fermi velocity. The present study has elucidated the dynamical properties of the $1 \mathrm{DES}$ in a dense metallic regime and provides valuable knowledge leading to the prospect of atomic-scale plasmonic device engineering (in the wide spectral region from terahertz to visible wavelengths) with also the spin degree of freedom.

This work has been supported by PRESTO and ICORP projects of Japan Science and Technology Agency.

*Electronic address: NAGAO.Tadaaki@nims.go.jp

[1] H. Raether, Excitation of Plasmons and Interband Transitions by Electrons (Springer-Verlag, Berlin, 1980).

[2] T. Nagao, T. Hildebrandt, M. Henzler, and S. Hasegawa, Phys. Rev. Lett. 86, 5747 (2001).

[3] T. Nagao, T. Hildebrandt, M. Henzler, and S. Hasegawa, Surf. Sci. 493, 680 (2001).

[4] P. Segovia, D. Purdie, M. Hengsberger, and Y. Baer, Nature (London) 402, 504 (1999).

[5] R. Losio, K. N. Altmann, A. Kirakosian, J.-L. Lin, D. Y. Petrovykh, and F. J. Himpsel, Phys. Rev. Lett. 86, 4632 (2001).

[6] K. N. Altmann, J. N. Crain, A. Kirakosian, J.-L. Lin, D. Y. Petrovykh, and F. J. Himpsel, Phys. Rev. B 64, 035406 (2001).

[7] J. N. Crain, J. L. McChesney, F. Zheng, M. C. Gallagher, P. C. Snijders, M. Bissen, C. Gundelach, S. C. Erwin, and F. J. Himpsel, Phys. Rev. B 69, 125401 (2004).

[8] J. R. Ahn, H. W. Yeom, H. S. Yoon, and I.-W. Lyo, Phys. Rev. Lett. 91, 196403 (2003).

[9] I. K. Robinson, P. A. Bennett, and F. J. Himpsel, Phys. Rev. Lett. 88, 096104 (2002).

[10] D. Sánchez-Portal, S. Riikonen, and R. Martin, Phys. Rev. Lett. 93, 146803 (2004).

[11] T. Nagao and S. Hasegawa, Surf. Interface Anal. 30, 488 (2000).

[12] T. Inaoka and T. Nagao (to be published).

[13] K. S. Singwi, M. Tosi, R. H. Land, and A. Sjölander, Phys. Rev. 176, 589 (1968); W. I. Friesen and B. Bergersen, J. Phys. C 13, 6627 (1980).

[14] We chose a constant value for $\varepsilon_{\mathrm{Si}}$ because, within the frequency range of our interest, it is nearly dispersionless $\left(\varepsilon_{\mathrm{Si}}=10.5-11.5\right)$, and the plasmon energy between the cases of constant $\varepsilon_{\mathrm{Si}}$ and the dispersing $\varepsilon_{\mathrm{Si}}$ differs by only a few percent over the entire dispersion curve.

[15] This value is the average of the $k_{F}$ values (0.38 and $0.44 \AA^{-1}$ ) of the two 1D bands (S1 and S2 bands in Fig. 3) reported in Ref. [6].

[16] The observed 1D plasmon can be attributed either to the individual excitations in both $\mathrm{S} 1$ and $\mathrm{S} 2$ bands or to the excitation only in the S1 band. Although it is not conclusive, the latter case seems to be more plausible, considering that the $\mathrm{S} 2$ band was recently reported to be insulating with a considerably large gap $(\sim 100 \mathrm{meV})[8]$.

[17] T. Inaoka, T. Nagao, S. Hasegawa, T. Hildebrandt, and M. Henzler, Phys. Rev. B 66, 245320 (2002).

[18] H. Totsuji, J. Phys. Soc. Jpn. 40, 857 (1976). 